\title{
Grafting of Silica Particles with Linoleic Acid via Modified Stober's Method for Preconcenrtration of Pesticides in Drinking Water
}

\author{
SADIA Atta ${ }^{1, a}$, MEHREEN Fatima ${ }^{1, b}$, ATIF Islam ${ }^{2, c, *}$, \\ NAFISA Gull, and MISBAH Sultan ${ }^{1, \mathrm{e}}$
}

${ }^{1}$ Institute of Chemistry, University of the Punjab, Lahore, 54590, Pakistan

${ }^{2}$ Department of Polymer Engineering and Technology,

University of the Punjab, Lahore, 54590, Pakistan

asad-ziam@yahoo.com, 'bmehreenfatima8914@gmail.com, 'dratifislam@gmail.com, nafisagull@gmail.com, ${ }^{e}$ misbahsultan@ymail.com

Keywords: Silica, Linoleic acid, Pesticides, HPLC, Solid Phase Extraction

\begin{abstract}
The extensive use of pesticides in agricultur has raised toxicity level in environment because of their mutagenic and carcinogenic activity. A sensitive technique is needed to develop for the detection of pesticides to get high sensitivity and selectivity in complex matrices. Modified Stober's method was used for the synthesis of pure $\mathrm{SiO}_{2}$ particles and grafted by linoleic acid. Grafted $\mathrm{SiO}_{2}$ were examined for its capability as a sorbent for removal and preconcentration of four organochlorine pesticides i.e. endrin, dieldrin, aldrin and DDT. The particles of $\mathrm{SiO}_{2}$ undergo hydrophobic interaction with linoleic acid by grafting. Both pure $\mathrm{SiO}_{2}$ and linoleic acid grafted $\mathrm{SiO}_{2}$ particles were characterized by SEM, FTIR and XRD. FTIR confirmed the grafting of $\mathrm{SiO}_{2}$ with linoleic acid. XRD and SEM results showed monodispersed and spherical $\mathrm{SiO}_{2}$ particles. The evaluation of grafted $\mathrm{SiO}_{2}$ was carried out by spiking organochlorine pesticides in drinking water. The grafted $\mathrm{SiO}_{2}$ particles could be used as a sorbent in solid phase extraction followed by high performance liquid chromatography with reverse phase $\mathrm{C} 18$ column with aqueous acetonitrile as a mobile phase with ultraviolet detector at $224 \mathrm{~nm}$. The percent recoveries for aldrin, dieldin, endrin and DDT were $81,52,60$ and $91 \%$, respectively.
\end{abstract}

\section{Introduction}

In recent years, grafted silica has stimulated the attention due to their extensive applications including drug delivery, catalysis, cosmetics, chromatography, agriculture and water purification. Silca appears to be a good medium for grafting as it is thermally and mechanically stable material. It is an inorganic polymer has poor interactions with non polar organic compounds Hence, silica particles are being loaded with linoleic acid through the process of grafting for separation and preconcentration of pesticides. Linoleic acid has got attention as organic modifier due to its properties of hydrophobicity, polar group, easy preparation and conjugated structure [1-3]. Organochlorine pesticides are the group of human-made pesticides which are cyclic polychlorinated molecules contain carbon, hydrogen and chlorine. They are dangerous as persistent in organically pollutant chemicals which break down very slowly and bioaccumulate in lipid rich tissues such as body fat [4-7].

The commonly used techniques for the detection of organochlorine pesticides are gas chromatography (GC) and high performance liquid chromatography (HPLC). Their direct determination in trace amount is difficult due to matrix interfences and insufficient detection power. They will undergo cleaning and enrichment step before analysis due to complexity of matrix and for accuracy [8]. A variety of enrichment techniques for pesticides from water have been used including liquid-liquid extraction (LLE), stir bar sorptive extraction (SBSE), liquid phase micro extraction (LPME), solid phase extraction (SPE) and solid phase micro exraction (SPME). SPE is being preferred as it has good recovery, availability of various sorbents, less use of organic solvents, less extraction time, ease of automation and operation [9-14]. The grafted materials are acquired by 
simple immobilization of an organic polymer or group on sized objects through covalent bond. The purpose of grafting is to enhance the selectivity of sorbent by developing interactions for particular analyte of interest [15-21].

In previous research works, silica was being modified with polymethyloctylsiloxane for preconcentration of pesticides with recovery of $72 \%$. Similiarly, $\mathrm{C}_{18}$ bonded silica was also used for extraction of pesticides from coconut water with the recovery of 74\% [22-23]. Carbon nanotubes recovered three toxic pesticides with $84-93 \%$ range [24]. Similarly, primary secondary amine was used as sorbent for preconcentration of twenty seven pesticides in radix astragali with recovery of $70-120 \%$ [25]. A polymeric resin abselut nexus was prepared as a sorbent to extract pesticides from water samples with recovery of 75\% [26]. The five SPE sorbent were prepared such as highly cross linked polystyrene divinylbenzene, $\mathrm{C}_{18}$ bonded silica, surface modified styrene divinylbenzene, divinylbenzene- $N$-vinylpyrrolidone and grafted carbon black for pesticides analysis with recovery of 70\% [27-28]. The application of grafted silica with linoleic acid in comparision to synthesized and commercially available silica appears to be more attractive in pesticides preconcetration due to its simple assembly, effectiveness and cost control operation. The reagent used for grafting should be non toxic and form strong interaction with pesticides [29-30].

In present study, novel SPE sorbent material has been prepared for preconcentration of pesticides in drinking water. Stober's method was modified and improved for synthesis of pure silica particles of different size. Linoleic acid (LA) was used as a modifier to be immobilize on the surface of silica particles. The grafted sorbent have characteristics of both silicaparticles (large surface area) and linoleic acid (hydrophobic and Van Der Waals interactions with given analytes). The modified silica particles and linoleic acid grafted silica particles were characterized by scanning electron microscope (SEM) analysis, fourier transform infrared spectroscopy (FTIR) and $\mathrm{X}$-ray diffraction. Grafted silica particles were used for the enrichment of pesticides and to carry out this. The newly prepared sorbent was packed in syringes and used in SPE. The capacity of grafted sorbent to retain pesticides was verified by HPLC with ultraviolet detection.

\section{Materials and Methods}

Materials. TCL Pesticides Mix standard was acquisited from Supelco (Bellefonte, PA, USA), Tetra ethoxy orthosilicate (molecular weight $=208.33 \mathrm{~g} / \mathrm{mol}$, analytical grade) was purchased from sigma Aldrichn, linoleic acid (molecular weight $=280.45 \mathrm{~g} / \mathrm{mol}$, analytical grade), ammonia, ethanol, $\mathrm{n}$ Hexane, deionized water and toluene was received from Merck. Methanol and acetonitrile of HPLC grade was purchased from sigma Aldrich. All chemicals were of analytical grade and used as received.

Method. In modified Stober's method, ethanol $(12 \mathrm{~mL})$, tetraethoxyorthosilicate $(6 \mathrm{~mL})$ and deionized water $(12 \mathrm{~mL})$ were taken in a conical flask and stirred continuously for $40 \mathrm{~min}$ on a hot plate. After that, ammonia $(9 \mathrm{~mL})$ was added and kept for stirring for $2 \mathrm{~h}$. Again after $2 \mathrm{~h}$ tetraethoxyorthosilicate $(3 \mathrm{~mL})$ was added upon stirring. Then, after $30 \mathrm{~min}$ ammonia $(9 \mathrm{~mL})$ was added and the reaction mixture was allowed to stir continuously for $24 \mathrm{~h}$. A white color solution was formed and it was centrifuged at $3000 \mathrm{rpm}$ for $20 \mathrm{~min}$. After centrifugation the resultant material was dried and as a result a pure white silica powder was formed. In this method instead of calcinations; centrifugation is being preferred as it provides extensive purification to silica particles. In addition to this for calcinations we have to heat our product which affects its further proceeding and its application.

Grafting of Linoleic Acid on the Surface of Silica. In this method, $100 \mathrm{~mL}$ of $\mathrm{n}$-hexane and $1.5 \mathrm{~g}$ of modified $\mathrm{SiO}_{2}$ particles were completely mixed in a three-necked round bottom flask outfitted with a thermometer and condenser, and then $0.5 \mathrm{~g}$ of linoleic acid was added. The mixture was heated under continuous stirring at $60{ }^{\circ} \mathrm{C}$ on hot plate for $4 \mathrm{~h}$ and then was cooled at $-5{ }^{\circ} \mathrm{C}$ for about $3 \mathrm{~h}$. After cooling, the mixture was centrifuged at $3000 \mathrm{rpm}$ for $20 \mathrm{~min}$ to obtain off-white colored precipitate. At the end, the precipitates were washed with the mixture of ethanol and deionized water in ratio of 3:7 by volume to obtain pure linoleic acid grafted silica particles. 


\section{Characterization}

Fourier Transform Infrared (FTIR) Spectroscopy. The spectra of synthesized modified silica and linoleic acid grafted modified silica $\left(\mathrm{SiO}_{2}-\mathrm{LA}\right)$ obtained from FTIR was examined using BRUKER model IFS 88 Infrared spectrophotometer in the range $4000-500 \mathrm{~cm}^{-1}$ on thin wafers of potassium bromide $(\mathrm{KBr})$ in which $1 \mathrm{wt} \%$ of synthesized materials were dispersed.

Scanning Electron Microscopy (SEM). Morphology of synthesized modified silica and linoleic acid grafted modified silica $\left(\mathrm{SiO}_{2}\right.$-LA) was determined by scanning electron microscope (SEM) photographs using a HITACHI S-4700 microscope performed at $60 \mathrm{kV}$. The samples were desiccated at $373 \mathrm{~K}$ for $240 \mathrm{~min}$ and layered with a coating before analysis.

X-ray Diffraction (XRD) analysis. XRD of modified silica and linoleic acid grafted modified silica $\left(\mathrm{SiO}_{2}\right.$-LA) was determined by using Rigaku D/Max $2500 \mathrm{VBZ}+/ \mathrm{PC}$ diffractometer, used $\mathrm{Cu}-$ $\mathrm{K} \alpha$ radiation $(40 \mathrm{kV}, 200 \mathrm{~mA})$ range between $3^{\circ}$ and $90^{\circ}, 2 \theta$ for wide angle range.

Stock Solution. Stock solution at the concentration of 200 ppm was prepared in n-hexane and stored in amber bottle at $4{ }^{\circ} \mathrm{C}$.

Primary Dilution Standard Solution. Working standard of 100 ppm was prepared from stock solution and diluted with n-Hexane (HPLC grade) by using Eq-1:

$$
\mathrm{C}_{1} \mathrm{~V}_{1}=\mathrm{C}_{2} \mathrm{~V}_{2}
$$

Spiked Solution. The spiked solution of $50 \mathrm{ppm}$ was prepared from primary dilution standard solution and diluted with distilled water upto mark.

\section{Application of Grafted Silica for Pesticides Preconcentration.}

Solid Phase Extraction. In a $3 \mathrm{~mL}$ disposable cartridge $200 \mathrm{mg}$ of linoleic acid grafted modified silica was compressed on a bottom. The sorbent was washed with methanol $(3 \mathrm{~mL})$ followed by hexane $(5 \mathrm{~mL})$. Subsequently the system was conditioned and activated with methanol $(3 \mathrm{~mL})$ and then by de ionized water $(5 \mathrm{~mL})$. A $50 \mathrm{~mL}$ of spiked water sample of pesticides were penetrated through the sorbent. The flow rate was adjusted about $2 \mathrm{~mL} / \mathrm{min}$ under vacuum system. After passing the whole sample through cartridge for enrichment step, it was dried for 10 min under vacuum to remove the solvent. Organochlorine compounds were restrained on the solid phase or sorbent and eluted with hexane $(5 \mathrm{~mL})$. The volume of eluate was decreased to $1 \mathrm{~mL}$ by using a gentle nitrogen stream.

High Performance Liquid Chromatography (HPLC). The HPLC was turned on half hour before the sample was injected. The sample was injected through $50 \mu \mathrm{L}$ micro-syringe. $20 \mu \mathrm{L}$ sample was enough to fill the injection loop and the extra sample went into the waste. The column was made of stainless steel, $25 \mathrm{~cm}$ in length, particle size $5 \mu \mathrm{m}$ and $4.6 \mathrm{~mm}$ internal diameter and column was made up of octadecyl silicon or $\mathrm{C}_{18}$ and HPLC with reverse phase mode was used. The solvent of acetonitrile and deionized water in ratio of 70:30 (v/v) were used as a mobile phase, isocratic elution for $5 \mathrm{~min}$ followed by linear gradient elution to $100 \%$ acetonitrile for $25 \mathrm{~min}$.The rate of flow of mobile phase was $1 \mathrm{~mL} / \mathrm{min}$. The back pressure of HPLC was 1400 psi. Detection was done by using UV-visible detector (Perkin Elmer 200) at wavelength $224 \mathrm{~nm}$. First the standard was run for 30 min to get standards chromatogram and then spiked pesticide water sample was under same conditions. The response of the chromatograph was sent to the computer and chromatogram was obtained by real time analysis. The formula used for calculation of percent recovery is described in Eq-2:

$$
\% \text { Recovery }=\frac{\text { Concentration after passing through SPE }}{\text { Known concentration of standard }} \times 100
$$

\section{Results and Discussion}

X-ray Diffraction Analysis. The synthesis of modified silica and its grafting with linoleic acid has confirmed by XRD are shown in Fig. 1. The chromatic intense beam of light falls on a parallel lattice plane of crystal, the incident beam is reflected secularly from various planes of crystal by process of crystal diffraction of scattering. The XRD results showed that high percentage of 
modified silica particles is amorphous but a few of them are crystalline. The major phase is $\mathrm{SiO}_{2}$ quartz and the minor phase is $\mathrm{SiO}_{2}$ cristobalite. The intense peak at $\theta=24^{\circ}$ showed that silica peak is amorphous in nature because of smaller particle size and incomplete inner structure of particles. No other peak other than silica showed purity of synthesized particles. The intensity of the diffraction peak is different in linoleic acid grafted silica particles which confirmed the grafting of linoleic acid on silica particles [31-32].

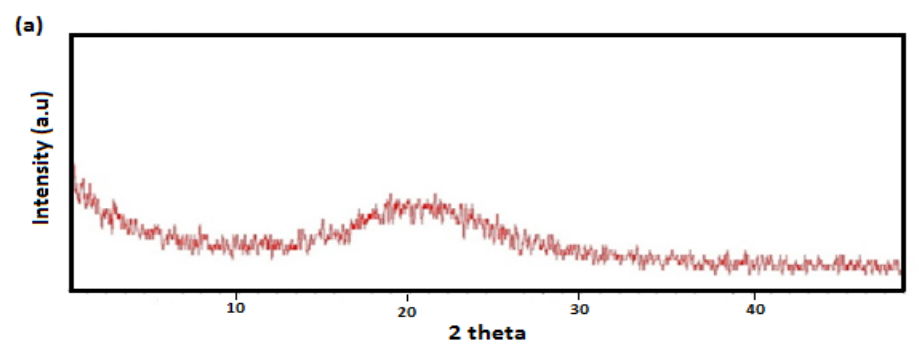

(b)

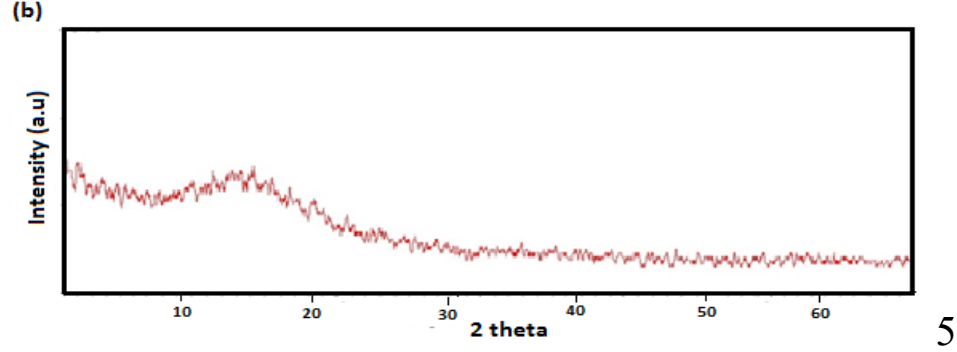

Fig. 1. X-ray diffraction pattern of (a) silica (b) Linoleic acid Grafted silica $\left(\mathrm{SiO}_{2}-\mathrm{LA}\right)$

Fourier Transform Infrared (FTIR) Analysis. Linoleic acid was selected for grafting on silica particles due to double bond carbonyl group along with a double bond in alkyl chain with a good hydrophobic, Van Der Waals and steric interactions with pesticides. Fourier transform infrared spectroscopy (FTIR) spectra of modified silica particles and linoleic acid grafted modified silica particles are shown in Fig. 2 respectively. In both FTIR spectra prominent peaks are appeared around $1090 \mathrm{~cm}^{-1}$ which coincide to stretching vibration of Si-O-Si together with peak at $810 \mathrm{~cm}^{-1}$ corresponds to symmetric vibration region of $\mathrm{Si}-\mathrm{O}-\mathrm{Si}$ groups results into synthesis of $\mathrm{SiO}_{2}$ particles. The high energy of Si-O bond provides silica with high thermal stability and low thermal expansion coefficient. In Fig. $2 b$ the peak of $\mathrm{COOH}$ which is characteristic of linoleic acid at $1729 \mathrm{~cm}^{-1}$ disappeared and a new peak at $1715 \mathrm{~cm}^{-1}$ coresponding to carboxylate appeared. It means $\mathrm{COOH}$ has reacted with $\mathrm{OH}$ group on the surface of silica and the product is carboxylate. The peak appeared in the region of $2800-2900 \mathrm{~cm}^{-1}$ corresponding to the absorption of the -CH symmetrical stretching vibrations because of attachment of linoleic acid group on surface of silica particles. This spectrum is obtained after sample washing with the mixture of ethanol and deionized water (3:7) by volume to remove unreacted linoleic acid in dried sample. It verified that the surface of modified silica particles has modified by linoleic acid through the reaction of functional sites of carboxylic acid with the Si-OH groups of silica particles [33-36].

Scanning Electron Microscopy (Morphological Analysis). $\mathrm{SiO}_{2}$ particles have high surface to volume ratio and small dimensions. In grafting process, linoleic acid is located within proximity of the material surface. Uniform spherical shaped $\mathrm{SiO}_{2}$ particles of size $884 \mathrm{~nm}$ was prepared by the modified Stober's method. The modified quantity of TEOS solution in ethanol affected monodispersity and size of particles. Scanning electron microscope (SEM), provide the direct evidence for existence of solid particles. They show homogeneous distribution of particles. It is observed that color and distribution of modified silica is quite different from that of linoleic acid grafted modified silica due to coating of linoleic acid on its surface. SEM analysis proved the capability of linoleic acid to react and grafting on the surface of modified silica by keeping its solid characters without solubilization. Scanning images of modified silica at high magnifications are the evidence of uniform distribution and homogeneous arrangement Fig. 3. 

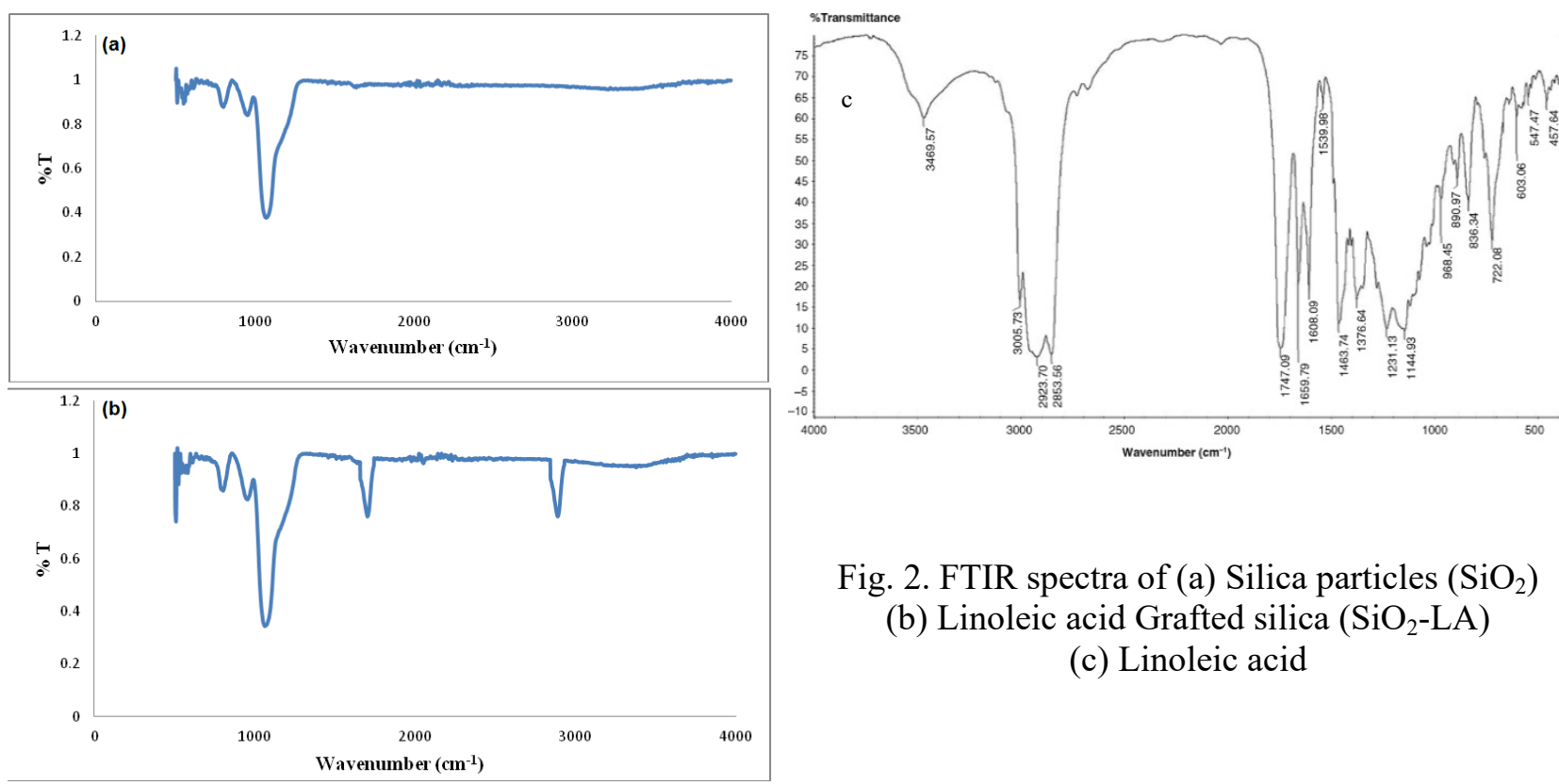

Fig. 2. FTIR spectra of (a) Silica particles $\left(\mathrm{SiO}_{2}\right)$

(b) Linoleic acid Grafted silica $\left(\mathrm{SiO}_{2}\right.$-LA)

(c) Linoleic acid
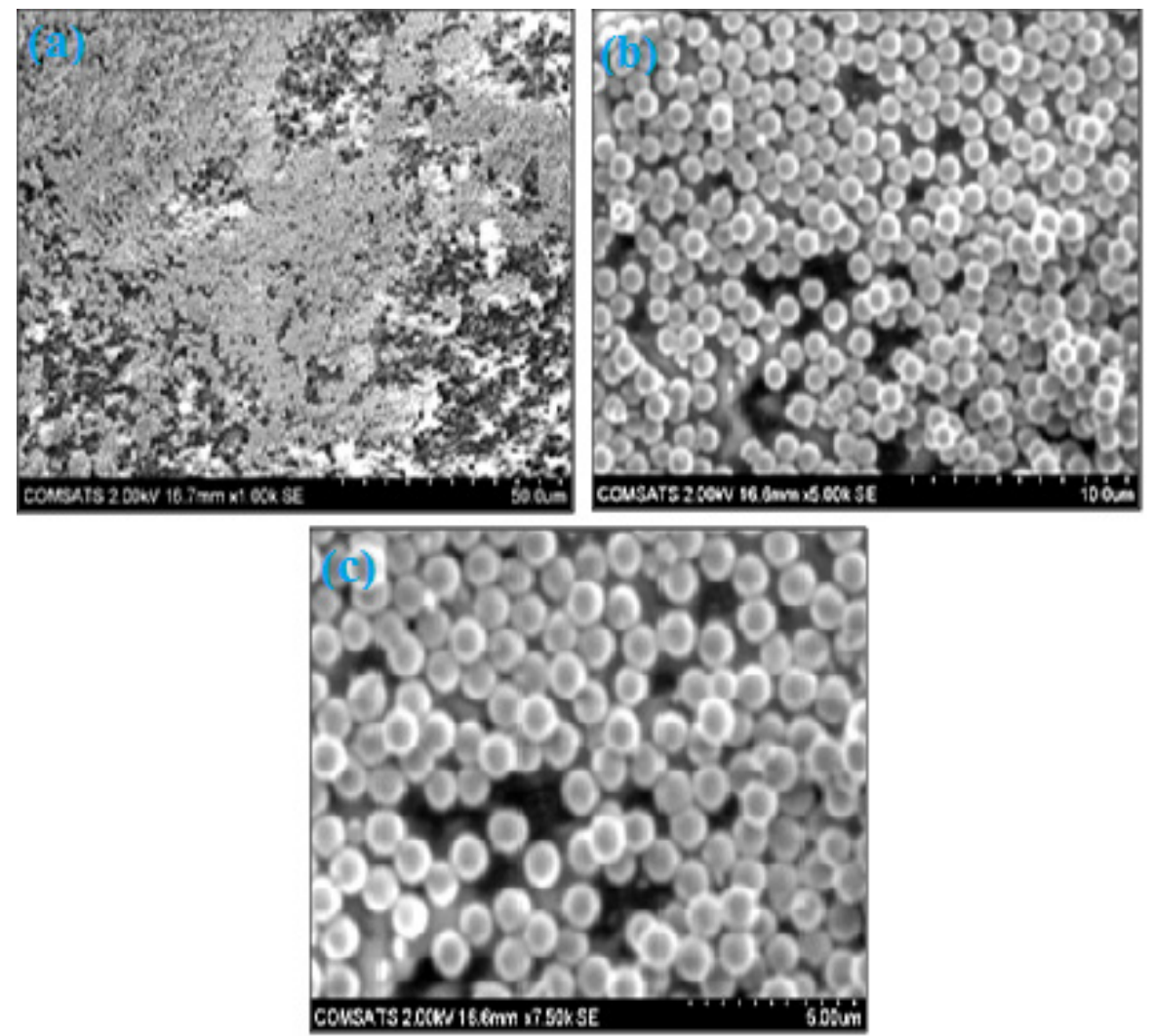

Fig. 3. SEM images of Silica particles (a) at magnificationof 1000x (b) at magnification of $5000 \mathrm{x}$ (c) at magification of $7500 \mathrm{x}$

Similarly SEM image of grafted modified silica at different magnifications prove that modified silica particles are covered with hydrophobic linoleic acid Fig. 3 [37, 38].

Evaluation of Method Performance. In HPLC analysis for pesticides, UV detector provide spectral information and is an easy way for the confirmation of the analyte components. The combination of C-18 type reversed stationary phase HPLC with UV detector prove to be capable of detecting a wide range of molecules and identify target compounds. The recovery of extracted target pesticides was determined by comparison of peak areas of chromatograms obtained from standard and spiked pesticide sample exhibited the ability of synthesized linoleic acid grafted modified silica $\left(\mathrm{SiO}_{2}\right.$-LA) sorbent to preconcentrate pesticides. The recovery was determined at 
the concentration of $50 \mathrm{ppm}$ and one obtained at the higher level using relative peak areas are shown in Table 1. The results showed that recoveries were greater than $80 \%$. Such high value of recoveries are due to a limited removal of the impurities from the analyte.

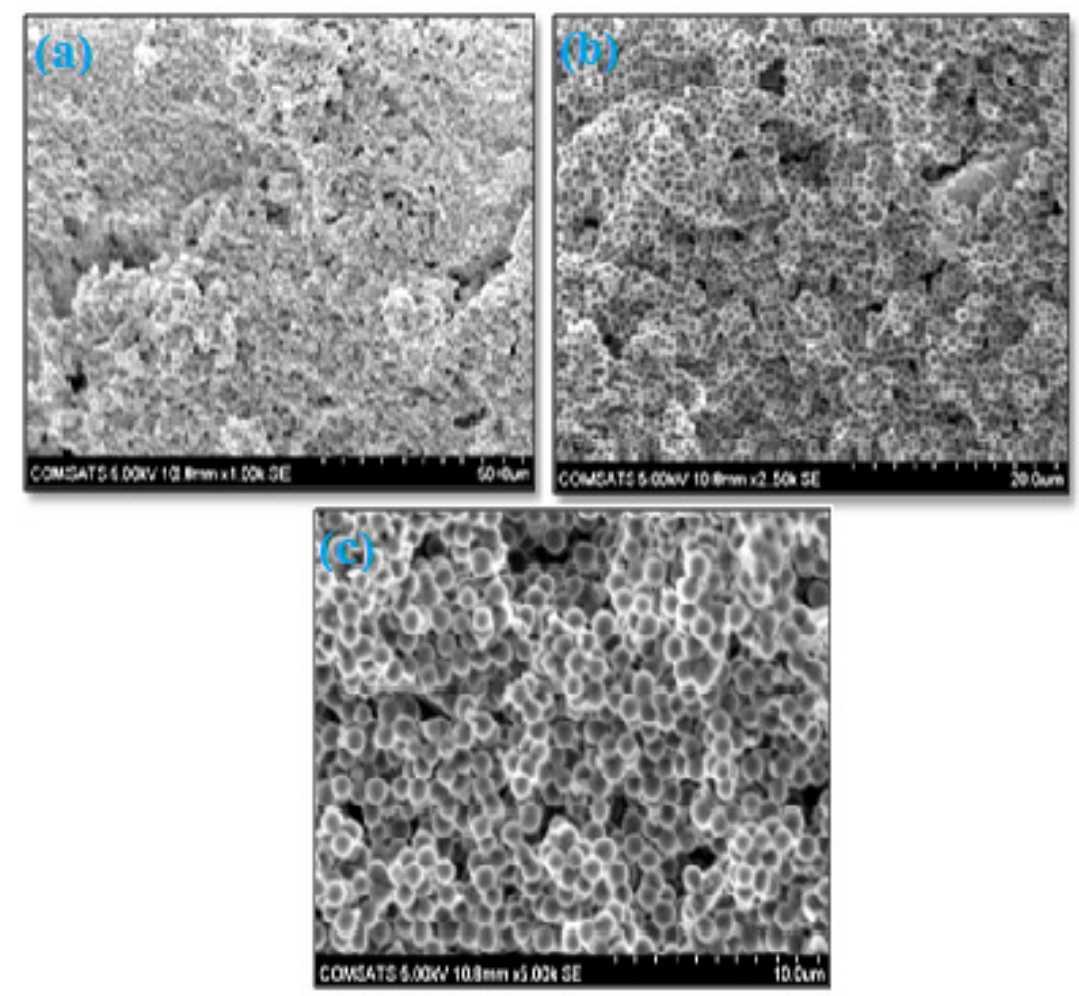

Fig. 4. SEM images of Linoleic acid Grafted Silica particles (a) at magnification of 1000x

(b) at magnification of $2500 \mathrm{x}$ (c) at magnification of $5000 \mathrm{x}$

Table 1. Percent recovery of pesticides using Linoleic acid grafted modified silica as a Sorbent

\begin{tabular}{ccccc}
\hline Sr. No. & Pesticides & $\begin{array}{l}\text { Volume of Eluent } \\
(\mathbf{m L})\end{array}$ & \%Recovery & $\begin{array}{c}\text { Stand. error the } \\
\text { mean }\end{array}$ \\
\hline 1 & Dieldrin & 5 & 52 & \\
2 & Endrin & 5 & 60 & 9.04 \\
3 & DDT & 5 & 91 & \\
4 & Aldrin & 5 & 81 & \\
\hline
\end{tabular}

Good recoveries were received successfully from newly made SPE sorbent obtained by grafting of linoleic acid on the modified silica, which was caused by the linoleic acid crosslinking, provided the excellent retention to an extraction phase. The proposed method for preconcentration of pesticides in this research work is directly compared with other previously reported methods, show that newly synthesized sorbent have good potential for pesticides extraction and reduces the toxic residues.

\section{Conclusions}

This research work demonstrated the direct extraction of pesticides from drinking water by using SPE-HPLC with good recoveries. The pure silica was synthesized by modified stober's method, both pure and grafted modified silica were characterized by SEM, FTIR and XRD. The results showed hydrophobic linoleic acid had ability to interact, bind and adsorb on the surface of modified silica sorbent. The extraction efficiency increased with the increase of the novel sorbent mass due to the increase of binding sites for the sorption. Linoleic acid grafted silica was evaluated for the extraction of wide chemical range of organochlorine pesticides. The modification in stober's method resulted into increase of particles size of $884 \mathrm{~nm}$. The newly synthesized linoleic acid 
grafted silica based sorbent had capability to retain and preconcentrate endrin, aldin, dieldrin and DDT pesticides with good recovery varied from 50 to $91 \%$. The results showed that solid phase extraction based technique with new sorbent can be a promising tool to improve the nowadays performance of methods used in pesticides control.

\section{Acknowledgements}

The author is highly obliged to the Institute of Chemistry, University of the Punjab, Lahore and PCSIR for providing lab facilities.

\section{References}

[1] B. Gorji, M.R. Allahgholi, R. Fazaeli, N. Niksirat, Synthesis and characterizations of silica nanoparticles by a new sol-gel method, J. Appl. Chem. Resear., 6 (2012) 22-26.

[2] I. Sowa, M.W. Kosior, K. Rokicka, R. Kocjan, G. Szymczak, Application of solid phase extraction with the use of silica modified with polyanilinefilm for pretreatment of samples from plant material before HPLC determination of triterpenic acids, Talanta 122 (2014) 51-57.

[3] V. Govindaraj, D. Mech, G. Pandey, R. Nagarajan, J.S.K. Sanwai, Kinetics of methane hydrate formation in the presence of activated carbon and nano-silica suspensions in pure water, J. Nat. Sci. Engineer., 26 (2015) 810-818.

[4] U. Ali, J.H. Syed, R.N. Malik, A. Katsoyiannis, J. Li, G. Zhang, C.K. Jones, Organochlorine pesticides (OCPs) in South Asian region: A review, Sci. Total Environ., 476-477 (2014) 705-717.

[5] L.A. Akkermans, J.D. Bercken, M.V. Helder, Excitatory and depressant effects of dieldrin and aldrin-transdiol in the spinal cord of the toad (Xenopus laevis). Eur. J. Pharmacol., 34 (1975) 133142.

[6] J. Michałowicz, K. Mokra, K. Rosiak, P. Sicinska, B. Bukowska, Chlorobenzenes, lindane and dieldrin induce apoptotic alterations in human peripheral blood lymphocytes (in vitro study), Environ. Toxicol. Pharmacol., 36 (2013) 979-988.

[7] A.H. Stern, Hazard identification of the potential for dieldrin carcinogenicity to humans. Environ. Res., 131 (2014) 188-214.

[8] M.H. Mashhadizadeh, M. Pesteh, M. Talakesh, I. Sheikhshoaie, M.M. Ardakani, M.A. Karimi, Solid phase extraction of copper (II) by sorption on octadecyl silica membrane disk modified with a new Schiff base and determination with atomic absorption spectrometry. Spectrochim. Act. B, 63 (2008) 885-888.

[9] G. Valencia, T.G. Llasera, Determination of organophosphorus pesticides in bovine tissue by an on-line coupled matrix solid-phase dispersion-solid phase extraction-high performance liquid chromatography with diode array detection method. J. Chromatogr. A, 39 (2011) 6869-6877.

[10] H. Farahani, Y. Yamini, S. Shariati, M.K. Zanjani, S.M. Baghahi, Development of liquid phase microextraction method based on solidification of floated organic drop for extraction and preconcentration of organochlorine pesticides in water samples, Anal. Chim. Act., 626 (2008) 166173.

[11] Y. Pico, M. Fernandez, M.J. Ruiz, G. Font, Current trends in solid-phase-based extraction techniques for the determination of pesticides in food and environment, J. Biochem. Biophys. Meth., 70 (2007) 117-131.

[12] L. Zhao, H.K. Lee, Application of static liquid-phase microextraction to the analysis of organochlorine pesticides in water, J. Chromatogr., A 919 (2001) 381-388. 
[13] A. Rodrigues, V. Ferreira, V.V. Cardoso, E. Ferreira, M.J. Benoliel, Determination of several pesticides in water by solid-phase extraction, liquid chromatography and electrospray tandem mass spectrometry, J. Chromatogr. A, 1150 (2007) 267-278.

[14] Momplaisira GM, Rosal CG, Heithmar EM, Varner KE, Riddick LA, Bradford DF, Halsell NT. Development of a solid phase extraction method for agricultural pesticides in large-volume water samples, Talanta., 81 (2010) 1380-1386.

[15] A.M. Geller, F.C. Stedile, M.R. Peralba, T.M. Pizzolato, J.D. Santos, Evaluation of zirconocene-based silica phases in organochloride pesticides preconcentration, J. Coll. Interf. Sci., 299 (2006) 163-171.

[16] X. Yue, S. Feng, S. Li, Y. Jing, C.. Shao, Bromopropyl functionalized silica nanofibers for effective removal of trace level dieldrin from water, Colloids Surf. A Physicochem. Eng. Asp., 406 (2012) 44-51.

[17] Q. Zhoua, Y. Gao, H. Bai, G. Xie, Preconcentration sensitive determination of pyrethroid insecticides in environmental water samples with solid phase extraction with $\mathrm{SiO}_{2}$ microspheres cartridge prior to high performance liquid chromatography, J. Chromatogr. A, 1217 (2010) 50215025.

[18] S. Chen, K. Sui, L. Chen, J.A. Pojman, Polyurethane nanosilica hybrid nanocomposites synthesized by frontal polymerization, J. Poly. Sci., 43 (2005) 1670-1680.

[19] A. Zhu, A. Cai, J. Zhang, H. Jia, J. Wang, PMMA-grafted-silica/PVC nanocomposites: Mechanical performance and barrier properties, J. Appl. Polym. Sci., 108 (2008) 2189-2196.

[20] C.S. Reddy, C.K. Das, M. Narkis, Propylene-ethylene copolymer nanocomposites: Epoxy resin grafted nanosilica as a reinforcing filler, Polym. Compos., 26 (2005) 806812.

[21] M.P. Sharma, G. Sadanandam, A. Ratnamala, V.D. Kumari, M. Subrahmanyam, An efficient and novel porous nanosilica supported $\mathrm{TiO}_{2}$ photocatalyst for pesticide degradation using solar light, J. Hazard. Mater., 171 (2009) 626-633.

[22] C.R.M. Vigna, L.S.R. Morais, C.H. Collins, I.C.S.F. Jardim, Poly(methyloctylsiloxane) immobilized on silia as a sorbent for solid phase extraction of some pesticides, J. Chromatogr. A, 1114 (2006) 211-215.

[23] L.S. Santos, N.S. Souza, J.A. Ferreira, A reversed-phase high-performance liquid chromatography method combined with matrix solid-phase dispersion extraction for the determination of teflubenzuron, lufenuron and bifenthrin residues in lyophilized coconut water, J. Food Comp. Anal., 26 (2012) 183-188.

[24] Y.A. Degs, M.A. Ghouti, A.E. Sheikh, Simultaneous determination of pesticides at trace levels in water using multiwalled carbon nanotubes as solid-phase extractant and multivariate calibration, J. Hazard. Mater., 169 (2009) 128-135.

[25] X. Mao, Y. Wan, A. Yan, M. Shen, Y. Wei, Simultaneous determination of organophosphorous, organochlorine, pyrethroid and carbamate pesticides in Radix astragali by microwave-assisted extraction/dispersive-solid phase extraction coupled with GC-MS, Talanta., 97 (2012) 131-141.

[26] G.M. Momplaisira, C.G. Rosal, E.M. Heithmar, K.E. Varner, L.A. Riddick, D.F. Bradford, N.T. Halsell, Development of a solid phase extraction method for agricultural pesticides in largevolume water samples. Talanta., 81 (2010) 1380-1386.

[27] D. Archivio, A.A. Fanelli, M. Mazzeo, P. Ruggieri, R. Fabrizio, Comparison of different sorbents for multiresidue solid-phase extraction of 16 pesticides from groundwater coupled with high-performance liquid chromatography, Talanta., 71 (2007) 25-30. 
[28] W. Huang, X.L. Fang, Z. Lu, Reinforcement of polysiloxane with superhydrophobic nanosilica, J. Mater. Sci., 44 (2004) 4522-4530.

[29] Z. Li, Y. Zhu, Surface-modification of $\mathrm{SiO}_{2}$ nanoparticles with oleic acid, Appl. Surf. Sci. 211 (2003) 315-320.

[30] K.W.J. Wahle, S.D. Heys, D. Rotondo, Conjugated linoleic acids: Are they beneficial or detrimental to health, Prog. Lipid Res., 43 (2004) 553-587.

[31] O.I. Lebedev, G.V. Tendeloo, O. Collart, P. Cool, E.F. Vansant, Structure and microstructure of nanoscale mesoporous silica spheres, Solid State Sci., 6 (2004) 489-498.

[32] H. Xu, F. Yan, E.E. Monson, R. Kopelman, Room temperature preparation and characterization of poly(ethyleneglycol)coated silica nanoparticles for biomedical applications, J. Biomed. Mater. Res. A, 66 (2003) 870-879.

[33] E. Grimvall, C.O. Stman, Retention characteristics of some selected halogenated environmental pollutants in silica and bonded normal phase liquid chromatography, J. Chromatogr. A, 675 (1995) 55-64.

[34] B. Aldona, S. Valdas, K. Marytè, J. Remigijus, K. Aivaras, FTIR, TEM and NMR investigations of Stöber Silica Nanoparticles, Mater. Sci., 10 (2004) 287-290.

[35] A.V. Helden, J.W. Jansen, A. Vrij, Preparation and characterization of spherical monodisperse silica dispersions in nonaqueous solvents, J. Colloid Interface Sci., 81 (1981) 354-368.

[36] K.S. Rao, K.E. Hami, T. Kodaki, K. Matsushige, K. Makino, A novel method for synthesis of silica nanoparticles, J. Colloid Interface Sci., 289 (2005) 125-131.

[37] G.H. Bogush, M.A. Tracy, C.F. Zukoski, Preparation of monodisperse silica particles: Control of size and mass fraction, J. Non-Cryst. Solid., 104 (1988) 95-106.

[38] I.D. Grice, M. Salzmann, I. Stiff, L.R. Griffith, Simultaneous determination of Aldrin, Dieldrin, Endrin, Heptachlor and p,p'-DDT in medicinal plant extracts using novel high performance liquid chromatography method, J. Liq. Chromatogr. Relat. Technol., 22 (1999) 23372344. 